Research Article

\title{
The Single-Vendor Multibuyer Integrated Production-Delivery Model with Production Capacity under Stochastic Lead Time Demand
}

\author{
Xuefang Sun \\ School of Economics and Management, Beihang University, Beijing 100191, China \\ Correspondence should be addressed to Xuefang Sun; sunxuefang1021@163.com
}

Received 10 October 2019; Revised 9 December 2019; Accepted 9 January 2020; Published 7 February 2020

Academic Editor: Kauko Leiviskä

Copyright ( $\odot 2020$ Xuefang Sun. This is an open access article distributed under the Creative Commons Attribution License, which permits unrestricted use, distribution, and reproduction in any medium, provided the original work is properly cited.

\begin{abstract}
In this paper, we consider an integrated production-delivery model in which a vendor supplies the same product to multiple buyers. Unlike existing study, in this proposed model, we assume that the sum of all buyers' demand rates is larger than the vendor's production rate under normal work, but less than that under overtime. All buyers are independent of each other. For each buyer, the lead time demand is stochastic and the shortage during lead time is permitted. The main objective of this model is to determine the optimal production and delivery policies and the optimal overtime strategy, which minimize the joint expected annual cost of the system. Based on the genetic algorithm, we develop a solution procedure to find the optimal production, delivery, and overtime decision of this model. Computational experiments show the error rate between the objective values obtained by the proposed solution procedure and the solutions solved by the exhaustive method. The results indicate that the proposed mixed genetic algorithm is more effective and adoptable in comparison with the exhaustive method as it can be able to calculate the optimal solutions for at least $96 \%$ for the instances. Ultimately, an adequate numerical example is given to show the detailed process of the solution procedure, and sensitivity analysis of main parameters with managerial implication is discussed.
\end{abstract}

\section{Introduction}

In the traditional supply chain system, management decisions for both the vendor and the buyer are independent of each other according to the classical economic production quantity (EPQ) or economic order quantity (EOQ) model. With the growing concentration on supply chain management, companies today are increasingly realizing that integration among supply chain's entities can gain more competitive advantages as this greatly saves overall supply chain costs. In the integrated supply chain system, all parties negotiate price, ordering, production, and other strategies. During last decades, the concept of integrated inventory management has attracted a great deal of attention of business managers and supply chain researchers (e.g., Yang and Wee [1]; Goyal and Gupta [2]; Viswanathan [3]; Bylka [4]; and Chan and Kingsman [5]).
In the traditional integrated production and delivery problem, it was assumed that market demand and lead time are constant and known. But in reality, it is impractical to have a fixed lead time for modern supply chains. Time, as a critical factor in commercial activities, not only causes the delivery delay but also decreases the stability and reliability of the whole system. Therefore, during the stochastic demand situation, lead time becomes an important issue. As Tersine [6] pointed out, lead time usually consists of order preparation, order transit, supplier lead time, delivery time, and setup time. In many practical situations, lead time is controllable; in other words, lead time can be shortened at the expense of additional costs. Therefore, managers can change the safety stock level, the loss of stock-out, and the customer service level by adjusting the lead time. Liao and Shyu [7] described that lead time consists of several components each having a different cost for reduced lead time. 
Zikopoulos [8] scripted an integrated inventory model taking stochastic lead time into consideration. Sarkar and Sarkar [9] assumed lead time being a linear function of three factors: batch size, setup time, and transportation time.

In real production activities, the vendor's production rate might not be large enough to satisfy the demand of customers owing to insufficient production capacity caused by being understaffed, incorrect decisions, possessing outdated equipment, and other restricted reasons. Managers usually adopt overtime strategies increasing the production rate to meet the market demand when limited production capacity exists. For example, Yamaha, as the largest manufacturer of musical instruments, establishes a regular overtime strategy during each production cycle because a rational and regular overtime schedule not only satisfies such large demand of customers but also avoids too high additional costs due to overtime. Many manufacturing enterprises, such as General Motors, Toyota, Ford, and Chrysler, take the same measure to cope with the fierce market competition. Liao and Huang [10], Dominguez et al. [11], and Zhang and Sun [12] studied overtime problem in an integrated model under capacity constraints. In this paper, we follow overtime strategy in the integrated model when the vendor's daily production rate is less than the daily demand rate.

The remainder of this paper is organized as follows: in Section 2, we present a literature review. In Section 3, we describe the related notations and assumptions used in the single-vendor multibuyer integrated model, and then in Section 4, we give a detailed description of this model and provide the joint expected annual cost function. We analyze propositions of the joint expected annual cost function and present a mixed genetic algorithm for finding the optimal production, shipment, and overtime policies in Section 5. In Section 6, computational experiments are conducted to show the effectiveness of the proposed algorithm. Finally, managerial implications and conclusions with some directions for further research are given in Sections 7 and 8.

\section{Literature Review}

The concept of the integrated inventory model for single vendor and single buyer was initialized by Goyal [13]. The framework he proposed is that the vendor provides a product made from an infinite production rate and ships to the buyer on the basis of a lot-for-lot policy. Banerjee [14] loosened the assumption of Goyal's [13] model and considered that the vendor produces for stock at a finite rate and delivers the whole batch to the retailer in the equal-size shipments. Under the fixed lot-size policy, Lu [15] further presented a one-vendor multibuyer integrated production-delivery model to coordinate the replenishment of different items. Hill [16] developed a more general integrated inventory model for determining the vendor's production quantity and successive shipment size. Huang [17] described an integrated vendorbuyer inventory model for items with imperfect quality. In his model, the delivery quantity depends on defective rate and batch size, and the delivery quantity from the vendor to the buyer is identical at every replenishment. Theoretical analysis shows that the expected integrated total cost function possesses convexities and that a solution procedure is derived to determine the optimal policy. Additionally, Huang [18] incorporated permissible delay in payments and order processing cost reduction into the integrated inventory model, and Lou and Wang [19] comprehensively extended the model of Huang [18] determining the optimal policies with ordering cost reduction. Under the determined market demand, Ouyang et al. [20] proposed an integrated inventory with capacity constraint and a permissible delay payment period that is order-size dependent. Lin et al. [21] formulated a generalized integrated inventory model to investigate the production and delivery policies while incorporating transportation time and capacity utilization. Other related research about integrated inventory model also can be found in the studies of Shahpouri et al. [22], Das et al. [23], Zhang and Sun [12], and Zhou and Wang [24].

For controllable lead time, Liao and Shyu [7] first presented an integrated inventory model which can be used to determine the length of lead time by minimizing the expected total relevant cost when the demand follows a normal distribution. Ben-Daya and Hariga [25] provided another integrated production inventory problem in which they relax the assumption of deterministic demand and assume that the lead time is varying linearly with the lot size. Pan and Yang [26] considered the lead time as a controllable factor in an integrated inventory model and estimated the lower joint total expected cost. Ouyang at el. [27] generalized Liao and Shyu's [7] model by permitting shortage and the lead time demand following a normal distribution and developed an iterative procedure for finding the optimal joint production and shipment policy. Recently, Lin [28] took controllable lead time into the integrated vendor-buyer inventory model to obtain the optimal production and shipment decisions by minimizing the joint expected total annual cost. Zikopoulos [8] considered controllable lead time in the integrated inventory model to maximize the system's profit. Sarkar and Sarkar [9] developed an integrated inventory model in which lead time is assumed to be a linear function of batch size, setup time, and transportation time. In their model, shortages are allowed not at the vendor but at the buyer, and shortages are partially backlogged with a backorder price discount.

Integrated inventory models with one vendor and multiple buyers have also been discussed by authors. Joglekar and Tharthare [29] built a more refined joint economic lot size model with one vendor and many identical buyers. Banerjee and Burton [30] described that all buyers adopt a common ordering cycle $T$ and that the vendor's production cycle is an integer multiple of $T$. Under integer-ratio policy, Abdul-Jalbar et al. [31] considered the shipments for the vendor being made before the whole lot produced, and they further provided a hypothetical condition that the replenishment interval for any buyer is greater than that for the vendor. Jha and Shanker 
[32] investigated a single-vendor multibuyer integrated model, in which each buyer's lead time demand is independently normally distributed and lead time can be reduced at an added crashed cost. Ben-Daya et al. [33] addressed an integrated inventory problem in the context of a closed-loop supply chain under a consignment stock. To clearly distinguish our model from the existing model, Table 1 shows a summary of the previous papers that are relevant to the integrated model for a vendor and multiple buyers.

In the traditional inventory model, the vendor's production capacity is infinite or larger than the market demand. However, in reality, many enterprises are suffering from limited production capacity. Overtime, as an effective measure, is commonly used in production activity. Bahl and Ritzman [34] initially researched the capacitated lot sizing problem by considering overtime into the model to change capacity. Adshead and Price [35] investigated the control of overtime in lot sizes from two aspects: the rate at which the level of overtime is adjusted in response to changes in the load on the shop (adjustment rate) and the basis on which the amount of overtime is set (setting rule). The related research about overtime problem can be referred to the studies of Liao and Huang [10], Dominguez et al. [11], and Lee and Kim [36]. Hariga [37] is one of the first researchers considering the overtime strategy into the model when limited production capacity exists. Recently, based on the model of Shu et al. [38], Zhang and Sun [12] took overtime into the single-vendor single-buyer integrated productiondelivery model under the vendor's limited production capacity. In their model, the vendor reserves a time interval to repair and maintain equipment and the transportation cost also cannot be neglected. Subsequently, Sun and Zhang [39] developed a single-manufacturer single-retailer integrated production-delivery model. In their model, the retailer's lead time demand is stochastic and the manufacturer adjusts the production rate through overtime when the production rate is smaller than the market demand.

In this paper, we extend Sun and Zhang's [39] model by considering that the vendor supplies the same product to multiple buyers. In this model, all buyers are independent of each other. The lead time demand of each buyer is stochastic and the lead time demand of each buyer follows a normal distribution. The vendor attempts to increase per unit output through overtime when the production rate is smaller than the total demand rates for all buyers. Meanwhile, from the view of costs for all buyers and the model, we follow the assumption of Abdul-Jalbar et al. [31] and assume that the length of vendor's production cycle is the maximum of ordering cycles for all buyers. The objective of the integrated model is to find the optimal delivery quantity, the length of overtime, reorder point, and lead time, which minimize the joint expected total cost of this two-stage supply chain system. Based on genetic algorithm, a solution procedure is developed and low error rate obtained from computational experiments shows the effectiveness of the proposed mixed genetic algorithm. Finally, a numerical example and sensitivity analysis of main parameters with managerial implication are discussed.

\section{Notations and Assumptions}

The following notations are used to develop a single-vendor multibuyer integrated model with limited production capacity under stochastic lead time demand.

\subsection{Notations}

$N$ : number of buyers

$D_{i}$ : average demand rate for buyer $i$ (units/year)

$T_{i}$ : ordering cycle for buyer $i$

$T$ : minimum value of ordering cycles for all buyers

$t$ : the length of overtime in a production cycle

$P$ : the vendor's production rate under normal work (units/year)

$(1+\alpha) P$ : the vendor's production rate under overtime, where $\alpha$ is the increasing rate for the production rate by overtime

$A_{i}$ : ordering cost per order for buyer $i$ ( $\$ /$ order)

$A_{v}$ : processing cost when the vendor accepts orders from buyers ( $\$ /$ process)

$h_{i}$ : unit holding cost for buyer $i$ ( $\$$ unit/year)

$h_{v}$ : unit holding cost for the vendor (\$/unit/year)

$Q_{i}$ : order quantity for buyer $i$ (units)

$R_{i}$ : reorder point for buyer $i$

$L_{i}$ : length of lead time for buyer $i$

$c$ : unit producing cost paid by the vendor under normal work (\$/unit)

$c_{1}$ : unit producing cost paid by the vendor under overtime (\$/unit)

$\pi_{i}$ : unit shortage cost for buyer $i$ (\$/unit/year)

$X_{i}$ : lead time demand for buyer $i$ which has a cumulative distribution function (c.d.f.) $F_{i}$ with finite mean $D_{i} L_{i}$ and standard deviation $\sigma \sqrt{L_{i}}$, where $\sigma$ denotes the standard deviation of the demand rate

$E(\cdot)$ : mathematical expectation

$x^{+}$: maximum value of $x$ and 0 , i.e., $x^{+}=\max \{x, 0\}$

$\mathrm{ETC}_{b}$ : expected annual cost for the buyer (\$/year)

$\mathrm{ETC}_{v}$ : expected annual cost for the vendor ( $\$ /$ year)

JETC: joint expected annual cost for the buyer and the vendor (\$/year)

Most of the following adopted assumptions are derived from the integrated production-delivery model of Sun and Zhang [39]:

(1) All buyers are independent of each other.

(2) Inventory is continuously reviewed. Buyer $i$ places the order when the on-hand inventory for buyer $i$ reaches the reorder point $R_{i}$. 
TABle 1: Comparison between this model and previous papers.

\begin{tabular}{lccc}
\hline Authors & Each buyer's demand rate & All buyers' ordering cycles & Vendor's production rate \\
\hline Joglekar and Tharthare [29] & Constant & Same & Infinite \\
Banerjee and Burton [30] & Constant & Same & Infinite \\
Lu [15] & Constant & Same & Infinite \\
Chan and Kingsman [5] & Constant & Different & Finite, larger than the average demand rate \\
Abdul-Jalbar et al. [31] & Constant & Different & Finite, larger than the sum of all buyers' demand rate \\
Jha and Shanker [32] & Stochastic & Same & Finite, larger than the sum of all buyers' demand rate \\
Ghiami and Williams [40] & Constant & Different & Finite, larger than the sum of all buyers' demand rate \\
Omar and Zulkipli [41] & Stock-dependent & Different & Finite, larger than the sum of all buyers' demand rate \\
Chan et al. [42] & Stochastic & Different & Finite, larger than the sum of all buyers' demand rate \\
Ben-Daya et al. [33] & Stochastic & Different & Finite, larger than the sum of all buyers' demand rate \\
Present model & Stochastic & Different & Finite, less than the sum of all buyers' demand rate \\
\hline
\end{tabular}

(3) Reorder point for buyer $i=$ the expected lead time demand + safety stock $\left(s_{i}\right)$, and $s_{i}=k_{i} \times$ standard deviation of lead time demand, i.e., $R_{i}=D_{i} L_{i}+$ $k_{i} \sigma \sqrt{L_{i}}$, where $k_{i}$ is a safety factor.

(4) To minimize the cost of this integrated model, the ordering point of each buyer should be integral multiples of $T$. The ordering cycle for buyer $i$ is $T_{i}=b_{i} T$, where $b_{i}$ is a positive integer.

(5) Shortage for each buyer is permitted.

(6) Referring to Abdul-Jalbar et al. [31], in view of the costs for buyers and the model, the length of the vendor's production cycle is maximum of ordering cycles for all buyers, i.e., the vendor's production cycle is $\max \left\{T_{1}, \ldots, T_{N}\right\}$.

(7) The transportation cost from the vendor to buyer $i$ is constant and independent of the ordering quantity. Hence, the transportation cost in this model is ignored.

\section{Integrated Production-Delivery Model}

In this section, we consider an integrated production-delivery model by considering that the vendor manufactures the same product for $N$ buyers in each production lot. The vendor manufactures the product with a limited production rate, and the production rate under normal work is less than the demand rate of all buyers, i.e., $P<\sum_{i=1}^{N} D_{i}$. For meeting the total demand of all buyers, the vendor adopts an overtime strategy to improve the output during each production cycle, i.e., $(1+\alpha) P \geq \sum_{i=1}^{N} D_{i}$.

For buyer $i$, when the inventory level drops to the point $R_{i}$, buyer $i$ reorders $Q_{i}$ unit products from the vendor. Since the expected lead time demand for buyer $i$ is $D_{i} L_{i}$, the expected inventory level before the receipt of the next order is $R_{i}-D_{i} L_{i}$. Furthermore, the expected annual inventory during each ordering cycle is $Q_{i} / 2+R_{i}-D_{i} L_{i}$. The expected annual holding cost for buyer $i$ can be formulated as $h_{i}\left(Q_{i} / 2+R_{i}-D_{i} L_{i}\right)$. In this integrated system, the length of lead time for buyer $i$ is $L_{i}$. The lead time demand $X_{i}$ for buyer $i$ is stochastic and has a cumulative distribution function (c.d.f.) $F_{i}$ with finite mean $D_{i} L_{i}$ and standard deviation $\sigma \sqrt{L_{i}}$. We follow the assumption that the reorder point for buyer $i=$ the expected lead time demand + safety stock, i.e., $R_{i}=D_{i} L_{i}+k_{i} \sigma \sqrt{L_{i}}$, where $k_{i}$ is a safety factor. When $X_{i}>R_{i}$, buyer $i$ is out of stock during the lead time $L_{i}$. Therefore, for buyer $i$, the expected shortage quantity can be expressed as $E\left(X_{i}-R_{i}\right)^{+}=\int_{R_{i}}^{+\infty}\left(x_{i}-R_{i}\right) \mathrm{d} F_{i}\left(x_{i}\right)$ and the expected annual shortage cost is $\pi_{i} E\left(X_{i}-R_{i}\right)^{+}$, where $\pi_{i}$ is the unit shortage cost for buyer $i$.

The minimization of the ordering cycles for all buyers is $T$. Following the assumption in Section 2, the ordering cycle for buyer $i$ is $T_{i}=b_{i} T$, where $b_{i}$ is a positive integer. The order quantity for buyer $i$ in each ordering cycle is $Q_{i}=D_{i} b_{i} T$. The ordering cost per order for buyer $i$ is $A_{i}$, and the expected annual ordering cost is $A_{i} / T_{i}=A_{i} /\left(b_{i} T\right)$. The expected annual cost for buyer $i$ can be expressed as

$$
\overline{\mathrm{TEC}}_{i}\left(b_{i}, T, k_{i}, L_{i}\right)=\frac{A_{i}}{b_{i} T}+h_{i}\left(\frac{D_{i} b_{i} T}{2}+k_{i} \sigma \sqrt{L_{i}}\right)+\pi_{i} E\left(X_{i}-D_{i} L_{i}-k_{i} \sigma \sqrt{L_{i}}\right)^{+}
$$

From the view of costs for buyers and the integrated model, we follow the hypothesis of Abdul-Jalbar et al. [31] and assume that the vendor's production process cycle is $\max \left\{T_{1}, \ldots, T_{N}\right\}$. Processing cost incurs when the vendor accepts orders from buyers. As described in notations, $A_{v}$ is the processing cost for the vendor and the expected annual processing cost is $A_{v} / T$. The expected production quantity for the vendor in each production cycle is

$$
\sum_{i=1}^{N} \frac{\max \left\{b_{1} T, \ldots, b_{N} T\right\}}{b_{i} T} Q_{i}=\max \left\{b_{1}, \ldots, b_{N}\right\} T \sum_{i=1}^{N} D_{i}
$$

Hence, it is easy to know that the vendor's expected annual holding quantity is $\left(\max \left\{b_{1}, \ldots, b_{N}\right\} T / 2\right) \sum_{i=1}^{N} D_{i}$. The vendor's unit holding cost is $h_{v}$, and the expected annual holding cost is given by $h_{v}\left(\max \left\{b_{1}, \ldots, b_{N}\right\} T / 2\right) \sum_{i=1}^{N} D_{i}$. 
As assumed above that the vendor's production rate is less than the sum of all buyers' demand rates under normal work, the vendor adopts an overtime strategy improving the vendor's outputs. To decrease the additional cost due to overtime, the production quantity should be equal to the total ordering quantity during a production cycle. As mentioned earlier, the vendor's expected production quantity during each production cycle can be expressed as

$$
\begin{array}{r}
\sum_{i=1}^{N} \frac{\max \left\{b_{1} T, \ldots, b_{N} T\right\}}{b_{i} T} Q_{i}=\max \left\{b_{1}, \ldots, b_{N}\right\} T \sum_{i=1}^{N} D_{i}, \\
(1+\alpha) P t+P\left[\max \left\{b_{1}, \ldots, b_{N}\right\} T-t\right]=\max \left\{b_{1}, \ldots, b_{N}\right\} T \sum_{i=1}^{N} D_{i} .
\end{array}
$$

The length of overtime for the vendor in a lot is $t=\left(\max \left(b_{1}, \ldots, b_{N}\right) T / \alpha P\right)\left(\sum_{i=1}^{N} D_{i}-P\right)$. However, the expected annual producing cost can be expressed as $\alpha c P+c_{1}(1+\alpha)\left(\sum_{i=1}^{N} D_{i}-P\right)-c\left(\sum_{i=1}^{N} D_{i}-P\right)$. For the sake of simplicity, let $b=\left(b_{1}, \ldots, b_{N}\right)$. Therefore, the vendor's expected annual cost is written as

$$
\begin{aligned}
\overline{\operatorname{TEC}_{v}}(T, b)= & \frac{A_{v}}{T}+\frac{h_{v} \max \left\{b_{1}, \ldots, b_{N}\right\} T}{2} \sum_{i=1}^{N} D_{i} \\
& +\alpha c P+c_{1}(1+\alpha)\left(\sum_{i=1}^{N} D_{i}-P\right)-c\left(\sum_{i=1}^{N} D_{i}-P\right) .
\end{aligned}
$$

And, the joint expected annual cost for the single-vendor multibuyer integrated model is

$$
\begin{aligned}
& \overline{J T C}_{v}\left(b, T, k_{1}, \ldots, k_{N}, L_{1}, \ldots, L_{N}\right) \\
& =\sum_{i=1}^{N}\left[\frac{A_{i}}{b_{i} T}+h_{i}\left(\frac{D_{i} b_{i} T}{2}+k_{i} \sigma \sqrt{L_{i}}\right)+\pi_{i} E\left(X_{i}-D_{i} L_{i}-k_{i} \sigma \sqrt{L_{i}}\right)^{+}\right] \\
& +\frac{A_{v}}{T}+\frac{h_{v} \max \left\{b_{1}, \ldots, b_{N}\right\} T}{2} \sum_{i=1}^{N} D_{i} \\
& +\alpha c P+c_{1}(1+\alpha)\left(\sum_{i=1}^{N} D_{i}-P\right)-c\left(\sum_{i=1}^{N} D_{i}-P\right) .
\end{aligned}
$$

Obviously, this is a mixed integer unconstrained minimization problem. To solve this problem, we analyze the mathematical properties of the expected annual cost function $\overline{\text { JTEC }}$ $\left(b_{1}, \ldots, b_{N}, T, k_{1}, \ldots, k_{N}, L_{1}, \ldots, L_{N}\right)$ in the next section.

\section{Solution Procedure}

5.1. Optimal Solution under Given b. The objective of this extended integrated model is to obtain the optimal strategy by minimizing the joint expected annual cost. Since the minimum of ordering cycles for all buyers is $T$, we can infer that at least one component of $b$ is 1 . For given $b$, we can derive that the cost function $\overline{\operatorname{JTEC}}\left(b, T, k_{1}, \ldots, k_{N}\right.$, $\left.L_{1}, \ldots, L_{N}\right)$ with respect to $T$ is convex. The optimal minimal ordering cycle $T$ is given in equation (6) by solving the first and second partial derivatives of $\overline{\operatorname{JTEC}}\left(b, T, k_{1}, \ldots\right.$, $\left.k_{N}, L_{1}, \ldots, L_{N}\right)$ with respect to $T$ as follows:

$$
T=\sqrt{\frac{2\left[A_{v}+\sum_{i=1}^{N} A_{i} / b_{i}\right]}{\sum_{i=1}^{N}\left[h_{i} b_{i}+h_{v} \max \left\{b_{1}, \ldots, b_{N}\right\}\right] D_{i}}} .
$$

For $k_{i}$, taking the first and second partial derivatives of $\overline{\operatorname{JTEC}}\left(b, T, k_{1}, \ldots, k_{N}, L_{1}, \ldots, L_{N}\right)$ with respect to $k_{i}$, we obtain

$$
\begin{aligned}
\frac{\partial \overline{\mathrm{TEC}_{v}}}{\partial k_{i}} & =\sigma h_{i} \sqrt{L_{i}}+\pi_{i}\left[F_{i}\left(D_{i} L_{i}+k_{i} \sigma \sqrt{L_{i}}\right) \sigma \sqrt{L_{i}}-\sigma \sqrt{L_{i}}\right], \\
\frac{\partial^{2} \overline{\operatorname{TEC}_{v}}}{\partial k_{i}^{2}} & =\pi_{i} f_{i}\left(D_{i} L_{i}+k_{i} \sigma \sqrt{L_{i}}\right) \sigma^{2} L_{i} .
\end{aligned}
$$

Obviously, $\quad\left(\partial^{2} \overline{\mathrm{JTEC}} / \partial k_{i}^{2}\right)>0 . \quad \overline{\mathrm{JTEC}}\left(b, T, k_{1}, \ldots, k_{N}\right.$, $\left.L_{1}, \ldots, L_{N}\right)$ with respect to $k_{i}$ is convex. Therefore, we obtain the optimal value of $k_{i}$ by setting $\left(\partial \overline{\mathrm{JTEC}} / \partial k_{i}\right)=0$.

$$
k_{i}=\max \left\{\frac{1}{\sigma \sqrt{L}_{i}} F^{-1}\left(1-\frac{h_{i}}{\pi_{i}}\right)-\frac{D_{i} \sqrt{L_{i}}}{\sigma}, 0\right\}, \quad i=1, \ldots, N,
$$

where $F^{-1}(\cdot)$ is the inverse function of cumulative distribution function (c.d.f.) $F$.

From equation (9), it can be concluded that the optimal values of each safety factor is independent of others and only hinge on the corresponding lead time $L_{i}$. Safety factor $k_{i}$ is a function with respect to $L_{i}$, and we denote $k_{i}$ as $k_{i}\left(L_{i}\right)$. For lead time $L_{i}$, we provide the following proposition.

Proposition 1. For given $b, \overline{J T E C}\left(b, T, k_{1}, \ldots, k_{N}, L_{1}, \ldots\right.$, $\left.L_{N}\right)$ is a convex function with respect to $L_{i}$ and the optimal lead time $L_{i}$ is

$$
L_{i}=\frac{F^{-1}\left(1-h_{i} / \pi_{i}\right)}{D_{i}}, \quad i=1, \ldots, N .
$$

Proof. See Appendix A for details.

Based on equations (6), (9), and (10), we obtain the production-delivery strategy and the corresponding expected annual cost JTEC for given $b$.

By analyzing the Proposition 1, we have the following lemma.

\section{Lemma 1}

(a) If $F^{-1}\left(\left(1-h_{i} / \pi_{i}\right) / D_{i}\right) \leq b_{i} T$, the length of lead time is larger than that of ordering cycle for buyer $i$

(b) If $F^{-1}\left(\left(1-h_{i} / \pi_{i}\right) / D_{i}\right)>b_{i} T$, the length of lead time is less than that of ordering cycle for buyer $i$

5.2. A Mixed Genetic Algorithm. In order to obtain the optimal solutions of the model in finite steps, we need to find out an effective solution procedure to give the optimal 
strategic decision. Proposition 2 shows the lower and upper bounds of each $b_{i}$.

Proposition 2. The component of $b$ and $b_{i}$ is an integer between 1 and

$$
\begin{array}{r}
\left\lfloor\frac{\left[\alpha c P+c_{1}(1+\alpha)\left(\sum_{i=1}^{N} D_{i}-P\right)-c\left(\sum_{i=1}^{N} D_{i}-P\right)\right]^{2}}{2 h_{v} A_{v} \sum_{i=1}^{N} D_{i}}-\frac{\sum_{i=1}^{N} A_{i}}{A_{v}}\right\rfloor \\
i=1, \ldots, N .
\end{array}
$$

Proof. See Appendix B for details.

For convenience, let

$$
b_{\max }=\left\lfloor\frac{\left[\alpha c P+c_{1}(1+\alpha)\left(\sum_{i=1}^{N} D_{i}-P\right)-c\left(\sum_{i=1}^{N} D_{i}-P\right)\right]^{2}}{2 h_{v} A_{v} \sum_{i=1}^{N} D_{i}}-\frac{\sum_{i=1}^{N} A_{i}}{A_{v}}\right\rfloor .
$$

When $b_{\max }$ and number of buyers $N$ are both small enough, we can obtain the corresponding expected annual cost JTEC by exhausting all feasible $b$. The minimum of these costs is regarded as the optimal expected annual cost $\overline{\mathrm{TEC}}^{*}$, and the corresponding solutions are the optimal solutions. However, when $b_{\max }$ or number of buyers $N$ is large enough, the exhaustive method is not a very effective approach. Hence, based on the detailed analysis above, we attempt to propose a solution procedure to search the global optimal solutions of the joint expected annual cost JTEC.

The genetic algorithm, invented by John Holland in 1962 , is a heuristic search process which is based on the rule of "survival of the fittest." It is commonly used in optimization problems, especially when the scale of solution is large. The starting step of the genetic algorithm is the creation of a population of biological units, which are called chromosomes. A chromosome further consists of smaller units called genes. In this model, we regard $b_{i}$ as a gene, and a chromosome composes of $N$ genes, $b_{1}, \ldots, b_{N}$. That is to say, we can treat any feasible $b$ as a chromosome and each chromosome is produced independently from others.

In this mixed integer unconstrained minimization problem, we give the definition of fitness for chromosome $j$ in population Pop as follows:

$$
\text { fitness }_{j}=\exp ^{-\left(f_{j}-f_{\min }\right)} \text {, }
$$

where $f_{\min }$ is the minimum of joint expected annual costs in population Pop and $f_{j}$ is the corresponding $\overline{\text { JTEC }}$ of chromosome $j$ in population Pop.

When the fitness of each chromosome $j$ in population Pop is determined, the probability of chromosome $j$ can be given to determine whether chromosome $j$ should be survived or rejected. Roulette wheel is a simply and common selection measure in optimization problems. In the integrated model, by adopting the roulette wheel selection of Lipowski and Lipowska [43], the probability of chromosome $j$ in population Pop can be calculated as

$$
\operatorname{prop}_{j}=\frac{\text { fitness }}{\sum_{z \in \text { Pop }_{\text {fitness }}}} \text {. }
$$

To avoid the unconstrained minimization problem plunging into the local optimal solutions, mutation is a very important operation. New genes of chromosome $j$ are produced via mutation, and we obtain new chromosomes to form a new population MutPop. The simplest and common way of implementing mutation is to mutate a gene of chromosome $j$ with an appropriate and low probability. In this model, the probability of gene $b_{i}$ on chromosome $j$ can be generated randomly.

Crossover is another measure of generating new chromosomes. After selection, the number of chromosomes in the new population becomes small. To keep the size of new population large enough, some new chromosomes are generated by using the one-point crossover and the twopoint crossover.

The detailed solution procedure to solve the mixed integer optimization problem is described as follows.

\subsection{Mixed Genetic Algorithm}

Step 1: give the number of iterations iter $_{\max }$ and the size of initial population NumPop.

Step 2: randomly generate an initial population Pop.

Step 3: let iter $:=1$, Pop (iter) $:=$ Pop and Min $:=+\infty$.

Step 4: for each chromosome $b$ in population Pop (iter), calculate the corresponding the joint expected annual cost JTEC combined with equations (6), (9), and (10). If $\overline{\mathrm{JTEC}}<$ Min, let Min $:=\overline{\mathrm{JTEC}}$.

Step 5: if iter $>$ iter $_{\max }$, stop. Otherwise, for each chromosome $b$ in population Pop(iter), calculate its fitness value combining with equation (11). Then, select some chromosomes having higher fitness values in population Pop(iter) to form a new population NewPop (iter + 1).

Step 6: for each chromosome $b$ in population NewPop $($ iter +1$)$, calculate its probability $\operatorname{prob}_{b}$ using equation (12). Then, select some chromosomes having lower probability values in population $\operatorname{NewPop}($ iter +1$)$ to form a new population NewPop1 (iter +1$)$.

Step 7: for each chromosome $b$ in population NewPop1 (iter +1$)$, randomly generate the probability rand of each gene on chromosome $b$. When rand is less than the given threshold, the value of the corresponding gene is randomly chosen from its interval. These new chromosomes form a new population MutPop (iter + 1).

Step 8: for all chromosomes in population MutPop $($ iter +1$)$, produce some chromosomes by randomly using crossover. Insert these chromosomes into population MutPop (iter +1 ), and obtain a new population CrossPop (iter +1$)$.

Step 9: let $\operatorname{Pop}($ iter +1$):=\operatorname{CrossPop}($ iter +1$)$ and iter $:=$ iter +1 ; then, go to Step 4 . 


\section{Computational Study}

The solution procedure of the mixed genetic algorithm described above is tested on 500 randomly generated test instances with $3,5,7$, and 9 buyers, respectively. In these instances, we set $D_{i} \in[35,43], A_{i} \in[20,50], h_{i} \in[0.005$, $0.015]$, and $h_{i} \in[0.02,0.03]$. To ensure that the solutions obtained by using the proposed mixed genetic algorithm are as close to the optimal solutions as possible, we give an appropriate number of initial population and a reasonable iterative number of the mixed genetic algorithm in each instance. Table 1 shows the computational results for various number of buyers in the single-vendor multibuyer integrated model. Here, the error rate is calculated as

$$
\text { error rate }=\frac{m_{\mathrm{mga}}}{m_{\text {total }}} \times 100 \%,
$$

where $m_{\text {mga }}$ is the total number of test instances obtained the optimal solution using proposed algorithm and $m_{\text {total }}$ is the total number of test instances.

In Table 2, it clearly demonstrates that the error rate is small enough to prove the effectiveness of the mixed genetic algorithm because this proposed solution procedure can be able to calculate the optimal solutions for at least $96 \%$ for the instances under varying number of buyers. Meanwhile, as can be observed in Table 2, the mixed genetic algorithm can effectively obtain the optimal solutions, and the solution procedure to a great extent can reduce the running time in comparison with the exhaustive method because this proposed algorithm's time complexity is $\mathrm{O}$ (iter $\times$ NumPop), while the exhaustive method's time complexity is $\mathrm{O}\left(b_{\max }^{N}\right)$.

To show the managerial and practical significance of the single-vendor multibuyer integrated model, we study a numerical example along with a data set. The parameters involved in the proposed model are assumed to take the values as given below: $P=100$ units/year, $A_{v}=100 \$ /$ setup, $h_{v}=0.015 \$ /$ unit/year, $c=3 \$ /$ unit, $c_{1}=4 \$ /$ unit, and $\alpha=$ 0.3 . Meanwhile, for each buyer $i$, we set $D_{i}=40$ units/year, $A_{i}=20 \$ /$ order, $h_{i}=0.005 \$ /$ unit/year, and $\pi_{i}=0.02 \$ /$ unit/ year. Figure 1 displays the expected annual cost of unit product with varying number of buyers in the extended integrated model. The expected annual cost of unit product is a convex with respect to the number of buyers. When $N=1$, the model in this paper becomes the single-vendor single-buyer integrated model of Banerjee [14]. However, this case can be analyzed by using the proposed iterative algorithm in the integrated model of Sun and Zhang [39] when the vendor's production rate is less than the buyer's demand. When $N=2$, the model is a simple single-vendor multibuyer integrated model and can be solved based on the analysis of Lu [15]. The vendor's production rate cannot meet the demand of all buyers when the number of buyers is larger than 2. Figure 1 depicts that it is beneficial to the integrated production-delivery model by adding several buyers properly and by reasonable overtime when the vendor has finite production capacity.

To further measure the parameters' influence on the overtime, production, and delivery policies of the integrated model, a numerical example with three identical buyers is
TABLE 2: Computational results with varying number of buyers.

\begin{tabular}{lcccc}
\hline Number of buyers & 3 & 5 & 7 & 9 \\
Error rate (\%) & 1.20 & 4.00 & 1.50 & 3.00 \\
\hline
\end{tabular}

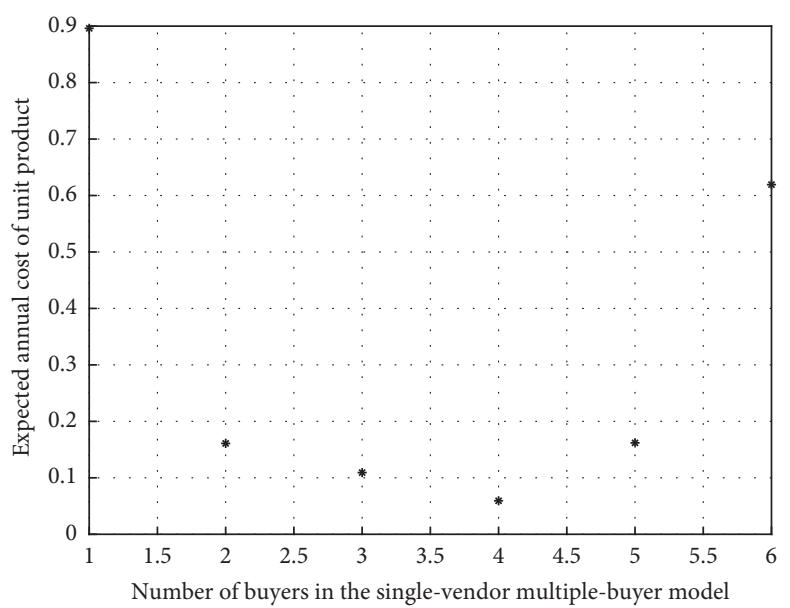

Figure 1: Expected annual cost of unit product with varying numbers of buyers.

conducted and $\alpha=0.25,0.30,0.35,0.40,0.45$. The values of other parameters follow the same given earlier.

Applying the proposed algorithm procedure yields the results as tabulated in Table 3. When the vendor has limited production capacity, it is apparent that the integrated system adds more cost when $\alpha$ increases. That is, when the vendor's production rate under normal work is less than the sum of all buyers' demand rates, the length of overtime in each production cycle shortens and the producing cost for the vendor adds as $\alpha$ increases.

Sensitivity analysis with respect to the key parameter, namely, $h_{i} / \pi_{i}$, was conducted as well. Figures 2 and 3 show the changed trend of lead time and safety factor for the buyer when $h_{i} / \pi_{i}$ varies. From Figure 2, the length of lead time decreases as $h_{i} / \pi_{i}$ increases. When $h_{i}=\pi_{i}$, the length of lead time for the buyer is 0 , i.e., the buyer has no lead time. When $h_{i}=0$, the length of lead time for the buyer is decided by the demand and its standard deviation. Figure 3 shows that the safety factor is a decreasing function with respect to $h_{i} / \pi_{i}$ in interval $[0,1]$. The buyer does not need safety stock when $h_{i} / \pi_{i}$ is larger than a certain number. In this case, when $h_{i} / \pi_{i}$ exceeds 0.5 , the reorder point for the buyer only is the expected lead time demand.

\section{Managerial Implication}

(i) This model has several managerial implications. In this model, it shows that properly adding the number of customers is beneficial for the enterprise' profit, when the enterprise's production capacity is finite. Hence, in reality, enterprise managers should attract a little more customers to order goods from the enterprise. In this situation, the enterprise's output per time is less than the market demand, and overtime is an effective measure to improve the 
TABLE 3: The optimal solutions under different values of $\alpha$.

\begin{tabular}{lcccccc}
\hline$\alpha$ & $t$ & $T$ & $k_{i}$ & $L_{i}$ & $Q_{i}$ & JTEC \\
\hline 0.25 & 4.4825 & 5.6032 & 0.6745 & 0.0199 & 224.1262 & 163.3520 \\
0.30 & 3.7354 & 5.6032 & 0.6745 & 0.0199 & 224.1262 & 182.3520 \\
0.35 & 3.2018 & 5.6032 & 0.6745 & 0.0199 & 224.1262 & 201.3520 \\
0.40 & 2.8016 & 5.6032 & 0.6745 & 0.0199 & 224.1262 & 220.3520 \\
0.45 & 2.4903 & 5.6032 & 0.6745 & 0.0199 & 224.1262 & 239.3520 \\
\hline
\end{tabular}

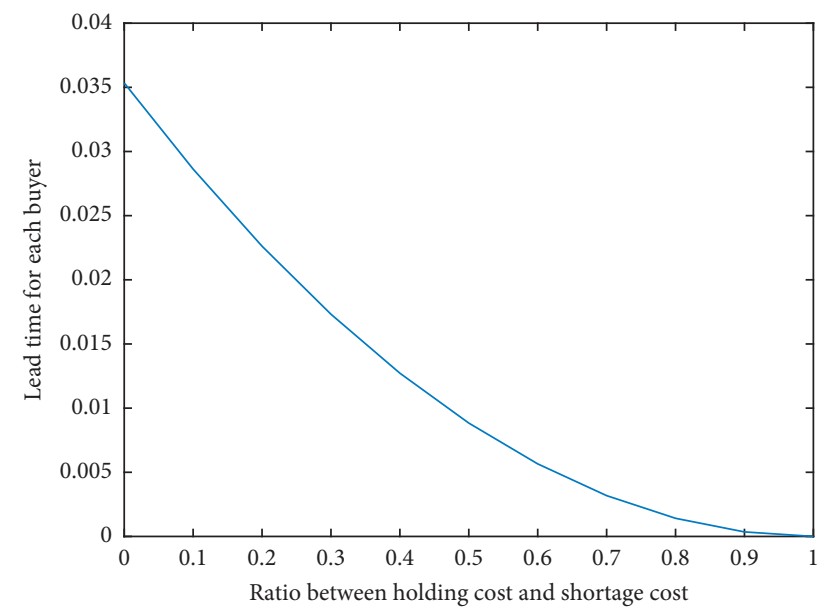

FIgURE 2: Impact of $h_{i} / \pi_{i}$ on the lead time for the buyer.

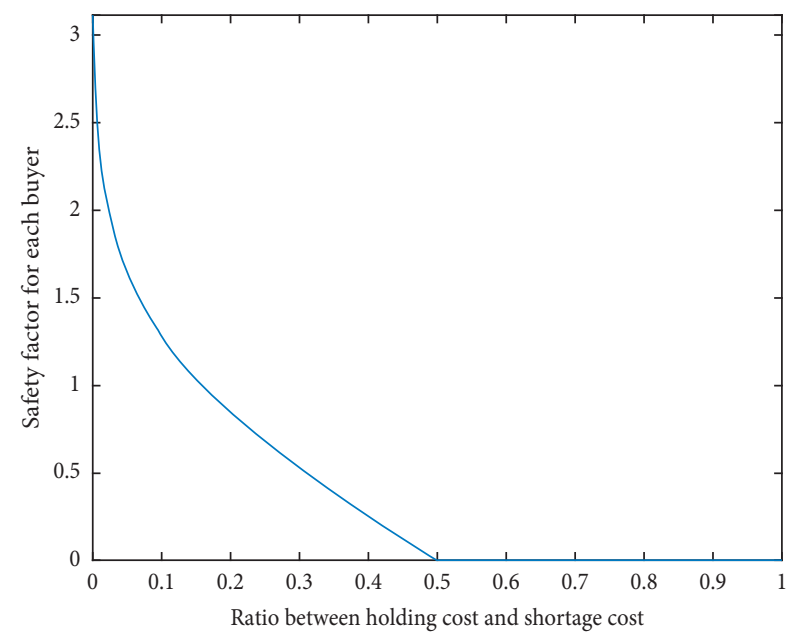

Figure 3: Impact of $h_{i} / \pi_{i}$ on the safety stock for the buyer.

production capacity and is commonly used in industrial activities.

(ii) On the other side, the proposed model adopts overtime strategy to improve the enterprise's output per time when the market demand exceeds production capacity. However, an unreasonable and inefficient overtime strategy not only inflates costs of the supply chain system but also wastes staff's offworking time. In this model, the cyclic and discontinuous overtime strategy helps the enterprise decrease holding cost to gain cost saving. At the same time, the staff of the manufacturing enterprise can set aside time for relaxation in long-working hours by adopting the proposed overtime policy. This overtime policy helps the enterprise achieve cost reduction and assists managers in configuring staff.

(iii) The introduced model decides how the parameters of the supply chain system impact the integrated system. As aforementioned discussion, it is clear that when the unit holding/shortage cost of each entity in the integrated model increases, so do the cost of the whole system. Meanwhile, the difference between unit holding cost and shortage cost for each buyer may decrease his/her lead time and safety stock. To cut down the total cost, each buyer can depend on not only decreasing the holding and shortage costs but also adjusting the ratio between these two parameters approaching to 1 . Thus, this model helps the buyers to appropriately store goods and coordinate with customers in order to adjust the unit holding and shortage costs.

\section{Conclusions}

In this paper, we consider an integrated production-delivery model in which a vendor supplies the same product to multiple buyers. Unlike existing vendor-buyer integrated models, we assume in this paper that the vendor's production rate is less than the sum of all buyers' demand rates under normal work. In order to increase the output, the vendor adopts overtime strategy to improve the vendor's production rate. Hence, the vendor's production rate under overtime is larger than the sum of all buyers' demand rates. Additionally, all buyers are independent of each other. The lead time demand for each buyer is stochastic and the shortage during lead time is permitted. To minimize the joint expected annual cost of the integrated system, we propose a mixed genetic algorithm to obtain the optimal production, delivery, and overtime policies of the system. Computational experiments show the error rate between the values obtained by the proposed solution and the solutions solved by the exhaustive method. The results indicate that the proposed mixed genetic algorithm is more effective and adoptable in comparison with the exhaustive method as it can be able to calculate the optimal solutions for at least $96 \%$ for the instances under varying number of buyers. Ultimately, an adequate numerical example is given to show the detailed process of the solution procedure, and sensitivity analysis is discussed.

There are a few limitations in this model. Firstly, in this model, the transportation cost is assumed to be constant and is ignored. However, the realistic transportation cost depends on truckload and the distance traveled by trucks. Secondly, fixed staff is mainly assumed in most literature. In the operation of enterprises, recruitment of new employees and departure of former employees are very common. This model can be extended to some more practical situations, such as considering the variable transportation cost and taking partially backlog and completely backlog into account. Another extension for the integrated system is taking staff changes and service-level constraints into consideration when overtime occurs. 


\section{Appendix}

\section{A. Proof of Proposition 1}

Since $T$ in equation (6) is fixed for given $b, \overline{\operatorname{JTEC}}(b, T$, $\left.k_{1}, \ldots, k_{N}, L_{1}, \ldots, L_{N}\right)$ can be rewritten as

$$
\overline{\operatorname{JTEC}}\left(b, T, k_{1}, \ldots, k_{N}, L_{1}, \ldots, L_{N}\right)=\sum_{i=1}^{N} J_{i}+X
$$

where

$$
\begin{aligned}
J_{i}= & h_{i} k_{i} \sigma \sqrt{L_{i}}+\pi_{i} E\left(X_{i}-D_{i} L_{i}-k_{i} \sigma \sqrt{L_{i}}\right)^{+}, \\
X= & \sum_{i=1}^{N}\left[\frac{A_{i}}{b_{i} T}+\frac{h_{i} D_{i} b_{i} T}{2}\right]+\frac{A_{v}}{T}+\frac{h_{v} \max \left\{b_{1}, \ldots, b_{N}\right\} T}{2} \\
& \cdot \sum_{i=1}^{N} D_{i}+\alpha c P+c_{1}(1+\alpha)\left(\sum_{i=1}^{N} D_{i}-P\right)-c\left(\sum_{i=1}^{N} D_{i}-P\right) .
\end{aligned}
$$

Obviously, $X$ is fixed for given $b$.

Combined with equation (9), and taking the first partial derivative of $\overline{\operatorname{JTEC}}\left(b, T, k_{1}, \ldots, k_{N}, L_{1}, \ldots, L_{N}\right)$ with respect to $L_{i}$, we obtain

$$
\frac{\partial \overline{\mathrm{JTEC}}}{\partial L_{i}}=\frac{\partial J_{i}}{\partial L_{i}}= \begin{cases}-h_{i} D_{i}<0, & L_{i}<F^{-1}\left(\frac{1-h_{i} / \pi_{i}}{D_{i}}\right), \\ D_{i} F\left(D_{i} L_{i}\right) \geq 0, & L_{i} \geq F^{-1}\left(\frac{1-h_{i} / \pi_{i}}{D_{i}}\right) .\end{cases}
$$

From the monotony of $\overline{\operatorname{JTEC}}\left(b, T, k_{1}, \ldots, k_{N}, L_{1}, \ldots\right.$, $\left.L_{N}\right)$ in $L_{i}$, for given $b$, $\overline{\operatorname{JTEC}}\left(b, T, k_{1}, \ldots, k_{N}, L_{1}, \ldots, L_{N}\right)$ is a convex function with respect to $L_{i}$ and the optimal lead time for buyer $i$ is

$$
L_{i}=F^{-1} \frac{1-h_{i} / \pi_{i}}{D_{i}}, \quad i=1, \ldots, N .
$$

\section{B. Proof of Proposition 2}

Suppose that the best inventory cost obtained currently is $\bar{C}$. The optimal inventory cost should not be larger than $\bar{C}$, i.e.,

$$
\begin{aligned}
& \sum_{i=1}^{N}\left[\frac{A_{i}}{b_{i} T}+h_{i}\left(\frac{D_{i} b_{i} T}{2}+k_{i} \sigma \sqrt{L_{i}}\right)+\pi_{i} E\left(X_{i}-D_{i} L_{i}-k_{i} \sigma \sqrt{L_{i}}\right)^{+}\right] \\
& +\frac{A_{v}}{T}+\frac{h_{v} \max \left\{b_{1}, \ldots, b_{N}\right\} T}{2} \sum_{i=1}^{N} D_{i} \\
& \quad+\alpha c P+c_{1}(1+\alpha)\left(\sum_{i=1}^{N} D_{i}-P\right)-c\left(\sum_{i=1}^{N} D_{i}-P\right) \leq \bar{C} .
\end{aligned}
$$

After some algebra, this yields

$$
\frac{\max \left\{b_{1}, \ldots, b_{N}\right\} \leq \bar{C}^{2}}{\left(2 h_{v} A_{v} \sum_{i=1}^{N} D_{i}\right)}-\frac{\left(\sum_{i=1}^{N} A_{i}\right)}{A_{v}} .
$$

In inequality (B.1), it is obviously known that $\bar{C} \geq \alpha c P+c_{1}(1+\alpha)\left(\sum_{i=1}^{N} D_{i}-P\right)-c\left(\sum_{i=1}^{N} D_{i}-P\right)$. Thus, we can further deduce

$$
\max \left\{b_{1}, \ldots, b_{N}\right\} \leq \frac{\left[\alpha c P+c_{1}(1+\alpha)\left(\sum_{i=1}^{N} D_{i}-P\right)-c\left(\sum_{i=1}^{N} D_{i}-P\right)\right]^{2}}{2 h_{v} A_{v} \sum_{i=1}^{N} D_{i}}-\frac{\sum_{i=1}^{N} A_{i}}{A_{v}}
$$

According to the mathematical property of $b_{i}$, $\{i=1, \ldots, N\}$, the following conclusion is obtained:

$$
\max \left\{b_{1}, \ldots, b_{N}\right\} \leq\left\lfloor\frac{\left[\alpha c P+c_{1}(1+\alpha)\left(\sum_{i=1}^{N} D_{i}-P\right)-c\left(\sum_{i=1}^{N} D_{i}-P\right)\right]^{2}}{2 h_{v} A_{v} \sum_{i=1}^{N} D_{i}}-\frac{\sum_{i=1}^{N} A_{i}}{A_{v}}\right\rfloor .
$$

Since $b_{i}$ is a positive integer, the lower and upper bounds of $b_{i}$ can be obtained as follows:

$$
1 \leq b_{i} \leq\left\lfloor\frac{\left[\alpha c P+c_{1}(1+\alpha)\left(\sum_{i=1}^{N} D_{i}-P\right)-c\left(\sum_{i=1}^{N} D_{i}-P\right)\right]^{2}}{2 h_{v} A_{v} \sum_{i=1}^{N} D_{i}}-\frac{\sum_{i=1}^{N} A_{i}}{A_{v}}\right\rfloor, \quad i=1, \ldots, N .
$$


Therefore, $b_{i}$ is an integer between 1 and

$$
\left\lfloor\frac{\left[\alpha c P+c_{1}(1+\alpha)\left(\sum_{i=1}^{N} D_{i}-P\right)-c\left(\sum_{i=1}^{N} D_{i}-P\right)\right]^{2}}{2 h_{v} A_{v} \sum_{i=1}^{N} D_{i}}-\frac{\sum_{i=1}^{N} A_{i}}{A_{v}}\right\rfloor, \quad i=1, \ldots, N
$$

\section{Data Availability}

No data were used to support this study.

\section{Conflicts of Interest}

The author declares no conflicts of interest.

\section{Acknowledgments}

This work was supported by the National Nature Science Foundation of China under Grants Nos. 71971010 and 71571006.

\section{References}

[1] P.-C. Yang and H.-M. Wee, "An arborescent inventory model in a supply chain system," Production Planning \& Control, vol. 12, no. 8, pp. 728-735, 2001.

[2] S. K. Goyal and Y. P. Gupta, "Integrated inventory models: the buyer-vendor coordination," European Journal of Operational Research, vol. 41, no. 3, pp. 261-269, 1989.

[3] S. Viswanathan, "Optimal strategy for the integrated vendorbuyer inventory model," European Journal of Operational Research, vol. 105, no. 1, pp. 38-42, 1998.

[4] S. Bylka, "Competitive and cooperative policies for the vendor -buyer system," International Journal of Production Economics, vol. 81-82, pp. 533-544, 2003.

[5] C. K. Chan and B. G. Kingsman, "Coordination in a singlevendor multi-buyer supply chain by synchronizing delivery and production cycles," Transportation Research Part E: Logistics and Transportation Review, vol. 43, no. 2, pp. 90-111, 2007.

[6] R. J. Tersine, Principles of Inventory and Materials Management, Prentice Hall, New York, NY, USA, 1994.

[7] C. J. Liao and C. H. Shyu, "An analytical determination of lead time with normal demand," International Journal of Operations \& Production Management, vol. 11, no. 9, pp. 72-78, 1991.

[8] C. Zikopoulos, "Remanufacturing lotsizing with stochastic lead-time resulting from stochastic quality of returns," International Journal of Production Research, vol. 55, no. 6, pp. 1565-1587, 2017.

[9] B. Sarkar and S. Sarkar, "An improved model with partial backlogging, time varying deterioration and stock-dependent demand," Economic Modelling, vol. 30, pp. 924-932, 2013.

[10] J.-J. Liao and K.-N. Huang, "Deterministic inventory model for deteriorating items with trade credit financing and capacity constraints," Computers \& Industrial Engineering, vol. 59, no. 4, pp. 611-618, 2010.

[11] R. Dominguez, B. Ponte, S. Cannella, and J. M. Framinan, "On the dynamics of closed-loop supply chains with capacity constraints," Computers \& Industrial Engineering, vol. 128, pp. 91-103, 2019.

[12] R. Q. Zhang and X. F. Sun, "Integrated production-delivery lot sizing model with limited production capacity and transportation cost considering overtime work and maintenance time," Mathematical Problems in Engineering, vol. 2018, Article ID 1569029, 10 pages, 2018.

[13] S. K. Goyal, "An integrated inventory model for a single supplier-single customer problem," International Journal of Production Research, vol. 15, no. 1, pp. 107-111, 1977.

[14] A. Banerjee, "A joint economic-lot-size model for purchaser and vendor," Decision Sciences, vol. 17, no. 3, pp. 292-311, 1986.

[15] L. Lu, "A one-vendor multi-buyer integrated inventory model," European Journal of Operational Research, vol. 81, no. 2, pp. 312-323, 1995.

[16] R. M. Hill, "The single-vendor single-buyer integrated production-inventory model with a generalized policy," European Journal of Operational Research, vol. 97, no. 3, pp. 493-499, 1997.

[17] C.-K. Huang, "An integrated vendor-buyer cooperative inventory model for items with imperfect quality," Production Planning \& Control, vol. 13, no. 4, pp. 355-361, 2002.

[18] C.-K. Huang, "An integrated inventory model under conditions of order processing cost reduction and permissible delay in payments," Applied Mathematical Modelling, vol. 34, no. 5, pp. 1352-1359, 2010.

[19] K.-R. Lou and W.-C. Wang, "A comprehensive extension of an integrated inventory model with ordering cost reduction and permissible delay in payments," Applied Mathematical Modelling, vol. 37, no. 7, pp. 4709-4716, 2013.

[20] L.-Y. Ouyang, C.-H. Ho, C.-H. Su, and C.-T. Yang, "An integrated inventory model with capapcity constraint and order-size dependent trade credit," Computers \& Industrial Engineering, vol. 84, pp. 133-143, 2015.

[21] F. Lin, T. Jia, F. Wu, and Z. Yang, "Impacts of two-stage deterioration on an integrated inventory model under trade credit and variable capacity utilization," European Journal of Operational Research, vol. 272, no. 1, pp. 219-234, 2019.

[22] S. Shahpouri, P. Fattahi, A. Arkan, and K. Parsa, "Integrated vendor-buyer cooperative inventory model with controllable lead time, ordering cost reduction, and service-level constraint," The International Journal of Advanced Manufacturing Technology, vol. 65, no. 5-8, pp. 657-666, 2013.

[23] B. C. Das, B. Das, and S. K. Mondal, "An integrated production-inventory model with defective item dependent stochastic credit period," Computers \& Industrial Engineering, vol. 110, pp. 255-263, 2017.

[24] Y.-W. Zhou and S.-D. Wang, "Optimal production and shipment models for a single-vendor-single-buyer integrated system," European Journal of Operational Research, vol. 180, no. 1, pp. 309-328, 2007.

[25] M. Ben-Daya and M. Hariga, "Integrated single vendor single buyer model with stochastic demand and variable lead time," International Journal of Production Economics, vol. 92, no. 1, pp. 75-80, 2004.

[26] J. C.-H. Pan and J.-S. Yang, "A study of an integrated inventory with controllable lead time," International Journal of Production Research, vol. 40, no. 5, pp. 1263-1273, 2002.

[27] L.-Y. Ouyang, K.-S. Wu, and C.-H. Ho, "Integrated vendorbuyer cooperative models with stochastic demand in 
controllable lead time," International Journal of Production Economics, vol. 92, no. 3, pp. 255-266, 2004.

[28] Y.-J. Lin, "An integrated vendor-buyer inventory model with backorder price discount and effective investment to reduce ordering cost," Computers \& Industrial Engineering, vol. 56, no. 4, pp. 1597-1606, 2009.

[29] P. Joglekar and S. Tharthare, "An individually responsible and rational decision approach to economic lot sizes for one vendor and many purchasers," Decision Sciences, vol. 21, no. 3, pp. 492-506, 1990.

[30] A. Banerjee and J. S. Burton, "Coordinated vs. independent inventory replenishment policies for a vendor and multiple buyers," International Journal of Production Economics, vol. 35, no. 1-3, pp. 215-222, 1994.

[31] B. Abdul-Jalbar, J. M. Gutiérrez, and J. Sicilia, "Policies for a single-vendor multi-buyer system with finite production rate," Decision Support Systems, vol. 46, no. 1, pp. 84-100, 2008.

[32] J. K. Jha and K. Shanker, "Single-vendor multi-buyer integrated production-inventory model with controllable lead time and service level constraints," Applied Mathematical Modelling, vol. 37, no. 4, pp. 1753-1767, 2013.

[33] M. Ben-Daya, R. As'ad, and K. A. Nabi, "A single-vendor multi-buyer production remanufacturing inventory system under a centralized consignment arrangement," Computers \& Industrial Engineering, vol. 135, pp. 10-27, 2019.

[34] H. C. Bahl and L. P. Ritzman, "A cyclical scheduling heuristic for lot sizing with capacity constraints," International Journal of Production Research, vol. 22, no. 5, pp. 791-800, 1984.

[35] N. S. Adshead and D. H. R. Price, "Overtime decision rule experiments with model of a real shop," European Journal of Operational Research, vol. 39, no. 3, pp. 274-283, 1989.

[36] Y. H. Lee and S. H. Kim, "Production-distribution planning in supply chain considering capacity constraints," Computers \& Industrial Engineering, vol. 43, no. 1-2, pp. 169-190, 2002.

[37] M. A. Hariga, "Economic production-ordering quantity models with limited production capacity," Production Planning \& Control, vol. 9, no. 7, pp. 671-674, 1998.

[38] M.-H. Shu, J.-C. Huang, and Y.-C. Fu, "A production-delivery lot sizing policy with stochastic delivery time and in consideration of transportation cost," Applied Mathematical Modelling, vol. 39, no. 10-11, pp. 2981-2993, 2015.

[39] X. Sun and R. Zhang, "The single-manufacturer single-retailer integrated production-delivery lot sizing model with production capacity under stochastic lead time demand," Procedia CIRP, vol. 83, pp. 528-533, 2019.

[40] Y. Ghiami and T. Williams, "A two-echelon production-inventory model for deteriorating items with multiple buyers," International Journal of Production Economics, vol. 159, pp. 233-240, 2015.

[41] M. Omar and H. Zulkipli, "A single-vendor multi-buyer integrated production-inventory system with stock-dependent demand," International Journal of Systems Science: Operations \& Logistics, vol. 5, no. 3, pp. 204-210, 2016.

[42] C. K. Chan, F. Fang, and A. Langevin, "Single-vendor multibuyer supply chain coordination with stochastic demand," International Journal of Production Economics, vol. 206, pp. 110-133, 2018.

[43] A. Lipowski and D. Lipowska, "Roulette-wheel selection via stochastic acceptance," Physica A: Statistical Mechanics and Its Applications, vol. 391, no. 6, pp. 2193-2196, 2012. 